\title{
Video Article \\ Isolation of Endocardial and Coronary Endothelial Cells from the Ventricular Free Wall of the Rat Heart
}

\author{
Alyssa Klein $^{1,2,3}$, Bethel Bayrau ${ }^{1,2,3}$, Yifei Miao ${ }^{1,2,3,4,5}$, Mingxia Gu $u^{1,2,3,4,5}$ \\ ${ }^{1}$ Department of Pediatrics, Division of Cardiology, Stanford School of Medicine \\ ${ }^{2}$ Vera Moulton Wall Center for Pulmonary Vascular Disease, Stanford School of Medicine \\ ${ }^{3}$ Stanford Cardiovascular Institute, Stanford School of Medicine \\ ${ }^{4}$ Division of Pulmonary Biology, Department of Pediatrics, Cincinnati Children's Hospital Medical Center \\ ${ }^{5}$ Center for Stem Cell and Organoid Medicine, CuSTOM, Division of Developmental Biology, and Perinatal Institute, Cincinnati Children's Hospital Medical Center
}

Correspondence to: Yifei Miao at yifei.miao@cchmc.org, Mingxia Gu at mingxia.gu@cchmc.org

URL: https://www.jove.com/video/61126

DOI: doi:10.3791/61126

Keywords: Developmental Biology, Issue 158, endocardium, coronary endothelium, isolation, heart, rat, CD31

Date Published: 4/15/2020

Citation: Klein, A., Bayrau, B., Miao, Y., Gu, M. Isolation of Endocardial and Coronary Endothelial Cells from the Ventricular Free Wall of the Rat Heart. J. Vis. Exp. (158), e61126, doi:10.3791/61126 (2020).

\section{Abstract}

It has been shown that endocardial endothelial cells (EECs) and coronary endothelial cells (CECs) differ in origin, development, markers, and functions. Consequently, these two cell populations play unique roles in cardiac diseases. Current studies involving isolated endothelial cells investigate cell populations consisting of both EECs and CECs. This protocol outlines a method to independently isolate these two cell populations for cell-specific characterization. Following the collection of the left and right ventricular free wall, endothelial cells from the outer surface and inner surface are separately liberated using a digestion buffer solution. The sequential digestion of the outer surface and the inner endocardial layer retained separation of the two endothelial cell populations. The separate isolation of EECs and CECs is further verified through the identification of markers specific to each population. Based on previously published single cell RNA profiling in the mouse heart, the Npr3, Hapln1, and Cdh11 gene expression is unique to EECs; while Fabp4, Mgll, and Cd36 gene expression is unique to CECs. qPCR data revealed enriched expression of these characteristic markers in their respective samples, indicating successful EEC and CEC isolation, as well as maintenance of cell phenotype, enabling further cell-specific functional analysis.

\section{Video Link}

The video component of this article can be found at https://www.jove.com/video/61126/

\section{Introduction}

This article provides a detailed protocol (modified from Gladka et al. ${ }^{1}$ ) for the dissection and subsequent isolation of endocardial endothelial cells (EECs) and coronary endothelial cells (CECs) from rat hearts. The ability to investigate these cell populations independently would enable the exploration of cell type-specific mechanisms underlying a variety of heart diseases that could serve as potential therapeutic targets. A successful method for the collection of these cell populations independently has yet to be published, however.

CECs differ from EECs in regards to their origin, markers, and functions during heart development and disease ${ }^{1,2,3,4,5,6,7}$. EECs stem from the ventral surface of the cardiac mesoderm ${ }^{3}$. They arise from Flk $1^{+}$progenitor cells in response to VEGF and HIF signaling and form the innermost layer of the three discrete regions of the developing heart: the atrium, ventricle, and sinus venosus ${ }^{3,6}$. Genetic lineage tracing suggests that the pluripotent endocardial cells of the sinus venosus derive venous cells, which migrate to form the subepicardial layer ${ }^{3}$. Subsequently, the subepicardial layer differentiates into coronary arteries and veins, including CECs, which remain across the peripheral ventricular free wall ${ }^{3,4}$. This endocardial to endothelial pathway is regulated by VEGFC, ELA/APJ, and SOX17 signaling ${ }^{3,4,6,8,9}$. The ventricular endocardium derives the fewer CECs of the interventricular septum by an unknown mechanism ${ }^{3}$. Subsequently, localized differentiation between EECs and CECs is suggested by markers specific to these two cell populations, including Mgll, Fabp4, and Cd36 expression in CECs, or Npr3, Cdh11, and Hapln1 expression in EECs ${ }^{3,5,10}$.

EECs and CECs play different roles in cardiac function. Endocardial to mesenchymal transition, valve formation, chamber maturation, outflow tract regulation, and atrioventricular canal development are contingent on EECs ${ }^{6}$. Alternatively, CECs contribute to vasomotor tone and inflammation of coronary arteries ${ }^{11}$. These variances in function result in individualized roles in disease development ${ }^{4,12}$. For instance, evidence suggests that malfunctioning EECs may lead to congenital valve disease ${ }^{6}$, noncompaction myocardium ${ }^{6}$, atrioventricular septal effect ${ }^{6}$, endocardial fibroelastosis ${ }^{13}$, hypoplastic left heart syndrome ${ }^{13}$, ventricular hypoplasia ${ }^{13}$, and cardiac hypertrophy ${ }^{12}$. Similarly, studies have found that abnormal CECs contribute to coronary artery disease ${ }^{14}$ and thrombosis ${ }^{11}$.

Successful isolation of EECs and CECs is necessary to achieve comprehensive knowledge of these two cell populations, which could be utilized in both the research and clinical settings. Determining the growth and differentiation factors of these cell populations would provide a reference 
for the differentiation of endothelial subtypes from induced pluripotent stem cells (iPSCs). Further, complete identification of the variances in the development, regulation, and function of EECs and CECs is vital for understanding the genomic and epigenomic factors responsible for numerous heart diseases in a cell type-specific manner. This article outlines steps for the successful collection of EECs and CECs independently, and provides evidence of separation by assessing the gene expression levels of cell type-specific markers.

Protocol

All animal procedures were approved by the Administrative Panel on Laboratory Animal Care (APLAC31608) at Stanford University.

\section{Preparation of buffers}

1. Prepare the digestion buffer using the reagents listed in Table 1.

1. Prepare the DNase I stock solution: Dissolve DNase I in RNase-free water for a stock solution of $2,000 \mathrm{units} / \mathrm{mL}(1 \mathrm{mg} / \mathrm{mL})$. Aliquot and store the stock solution at $-20^{\circ} \mathrm{C}$.

2. Prepare the liberase solution: Dissolve liberase with RNase-free water for a stock solution of $5 \mathrm{mg} / \mathrm{mL}$. Rotate it on a roller bank at $4{ }^{\circ} \mathrm{C}$ for $30 \mathrm{~min}$. Aliquot and store the stock solution at $-20{ }^{\circ} \mathrm{C}$.

2. Prepare the sorting buffer by diluting $0.5 \mathrm{M}$ ethylenediaminetetraacetic acid (EDTA) and $10 \%$ bovine serum albumin (BSA) stock solution with $1 \mathrm{x}$ phosphate buffered saline (PBS) for a final concentration of $2 \mathrm{mM}$ of EDTA and $0.5 \%$ of BSA.

\section{Heart collection}

NOTE: Six Sprague Dawley rats weighing 50-100 g were used in this protocol. No gender difference was observed.

1. Euthanize the rat with $\mathrm{CO}_{2}$ and place it in a supine position on a dissecting platform. Pin the extremities down and sterilize both the abdomen and chest with $70 \%$ ethanol.

2. Open the abdomen with scissors and insert a $21 \mathrm{G}$ needle into the portion of the posterior vena cava located behind the intestines. Pull the syringe plunger back, withdrawing the blood from the vein until the liver appears lighter in color and no more blood can be withdrawn, indicating sufficient blood removal.

3. Using scissors, open the chest carefully to avoid damaging the lung and the heart. Lift the aorta arch/atrium with the forceps and cut the aorta, pulmonary artery, pulmonary veins, and vena cava to liberate and remove the heart.

4. Wash the heart with $50 \mathrm{~mL}$ of cold $1 x$ Hanks' balanced salt solution (HBSS) buffer $3 x$ to remove excessive blood.

5. Under a microdissection microscope, remove the right ventricular free wall into a $5 \mathrm{~mL}$ tube containing Dulbecco's modified Eagle medium (DMEM) (Figure 1A,B).

1. Lay the heart on its flatter, posterior face to identify the left and right side.

2. Locate the pulmonary artery, the most anterior of the veins and arteries branching from the top of the heart and cut through the pulmonary artery down to the right ventricular chamber. On the anterior face of the heart, cut along the septum until reaching the apex. Continue cutting from the apex up the posterior side of the heart along the septum until the pulmonary artery and right ventricular chamber junction point (Figure 1A).

3. Starting from the pulmonary artery and right ventricular junction point, cut both the anterior and posterior side of the heart perpendicularly to the previous dissection and away from the septum, until the right ventricular free wall is liberated from the rest of the heart (Figure 1B).

6. Under the microdissection microscope, remove the left ventricular free wall into a $5 \mathrm{~mL}$ tube containing DMEM (Figure 1C,D).

1. Locate the aorta, branching from the top of the heart behind the pulmonary artery, and repeat the method described in step 2.5 to cut off the left ventricular free wall.

\section{EEC digestion}

1. Place both right and left ventricular free wall tissues in a $60 \mathrm{~cm}$ culture dish with the inner surface lying flat, facing down (Figure 2). NOTE: The inner surface is the interior face of the slightly concave shaped ventricle as shown in Figure 2A,C.

2. Add $0.5-1 \mathrm{~mL}$ of digestion buffer to the dish, placing the tip of the pipette directly under the tissue. Continue until only the inner surface is immersed.

3. Incubate the dish in a $5 \% \mathrm{CO}_{2}, 37^{\circ} \mathrm{C}$ incubator for $5 \mathrm{~min}$.

4. Add EC medium to the culture dish to stop the digestion. NOTE: Keep the amount of digestion buffer to EC medium as a 1:5 ratio.

5. Use a $1 \mathrm{~mL}$ pipette to flush the inner surface of the ventricles with $\mathrm{EC}$ medium and transfer the runoff into a $50 \mathrm{~mL}$ collection tube through a $40 \mathrm{~mm}$ strainer (Figure 2E). Keep the collections on ice for downstream purification.

\section{CEC digestion}

1. Cut along the outer surface of the left ventricle without contamination from the inner layer (Figure 2B,D) and place each in a separate $5 \mathrm{~mL}$ tube containing $1 \mathrm{~mL}$ of the digestion buffer.

NOTE: The left ventricle can be identified as the thicker ventricle, and the outer surface can be determined as the exterior face of the slightly concave ventricle.

2. Using dissection scissors, mince the ventricular wall into small, $1 \mathrm{~mm}^{3}$ pieces. 
3. Incubate the tube in a $37^{\circ} \mathrm{C}$ water bath for about $15-20 \mathrm{~min}$. Vortex every $2-3 \mathrm{~min}$.

4. Pipette $4 \mathrm{~mL}$ of EC medium into the $5 \mathrm{~mL}$ tube to terminate the digestion.

5. Transfer the solution with a $5 \mathrm{~mL}$ serological pipette into a $50 \mathrm{~mL}$ tube through a $40 \mathrm{~mm}$ strainer (Figure $2 \mathrm{E}$ ). Keep the collections on ice for downstream purification.

\section{Cell collection}

1. Centrifuge the tubes at $300 \times g$ for $10 \mathrm{~min}$ at room temperature (RT) and then aspirate the supernatant.

2. If the pellet appears red, resuspend the pellet in 1-2 $\mathrm{mL}$ of $1 \mathrm{x}$ red blood cell (RBC) lysing buffer (Figure 2F). NOTE: If there are no RBCs, proceed to step 6.

3. Incubate the tubes in a $37^{\circ} \mathrm{C}$ water bath for $5 \mathrm{~min}$.

4. Pipette $10 \mathrm{~mL}$ of PBS into the $50 \mathrm{~mL}$ tubes to stop the lysis.

5. Centrifuge the tubes at $300 \times g$ for $10 \mathrm{~min}$ at RT, and then aspirate the supernatant.

\section{EC sorting}

1. Add $90 \mu \mathrm{L}$ of sorting buffer and $10 \mu \mathrm{L}$ of anti-CD31 PE antibody into the $50 \mathrm{~mL}$ tubes.

2. Vortex the tubes and then incubate them for $10 \mathrm{~min}$ in a $4{ }^{\circ} \mathrm{C}$ fridge.

3. Add $10 \mathrm{~mL}$ of sorting buffer into the tubes, mix thoroughly, and then centrifuge at $300 \times \mathrm{g}$ for $10 \mathrm{~min}$ at RT.

4. Aspirate the supernatant and resuspend the pellet in $80 \mu \mathrm{L}$ of sorting buffer and $20 \mu \mathrm{L}$ of anti-PE microbeads.

5. Vortex the tubes and incubate at $4{ }^{\circ} \mathrm{C}$ for $15 \mathrm{~min}$.

6. Wash the cells by adding $10 \mathrm{~mL}$ of sorting buffer into the tubes, mix thoroughly, and then centrifuge them at $300 \times \mathrm{g}$ for $10 \mathrm{~min}$ at RT.

7. Aspirate the supernatant and resuspend the pellet in $500 \mu \mathrm{L}$ of sorting buffer.

8. Place a column containing superparamagnetic spheres into a magnetic separator and rinse the column with $3 \mathrm{~mL}$ of sorting buffer.

9. After the column are "flow stop", pipette $500 \mu \mathrm{L}$ of the cell suspension into the columns and then wash the columns with sorting buffer. Repeat the wash $3 \mathrm{x}$, each with $3 \mathrm{~mL}$ of sorting buffer (Figure 2G).

10. Remove the columns from the separator and place them on top of a new $15 \mathrm{~mL}$ collection tube.

11. With a pipette, add $5 \mathrm{~mL}$ of EC medium to the columns, and propel the solution through using a column plunger.

12. Centrifuge the collection tubes at $300 \times g$ for $10 \mathrm{~min}$ at RT and then aspirate the supernatant.

13. Extract the RNA from the cell pellet of the EEC and CEC samples using a kit, following the manufacturer's instructions. A minimum of 500 ng of RNA can be obtained. NOTE: After RNA extraction, the sample can be stored at $-80^{\circ} \mathrm{C}$.

14. Reverse transcribe $500 \mathrm{ng}$ of RNA to get cDNA using a random primer and a kit, following the manufacturer's instructions.

15. Perform quantitative PCR for the validation of endocardial and endothelial populations (Figure $2 \mathrm{H}$ ). Primer sequences are listed in Table 2.

1. Add $5 \mu \mathrm{L}$ of EEC or CEC cDNA (1 ng/ $\mu \mathrm{L})$ into the wells of a $384 \mathrm{qPCR}$ plate. Add the EEC or CEC cDNA into enough wells so that there are three wells per number of primers.

2. Mix $6 \mu \mathrm{L}$ of $2 \times$ qPCR SYBR green buffer with $0.5 \mu \mathrm{L}$ of each $10 \mu \mathrm{M}$ primer, including both forward and reverse primers, and add them into a single well corresponding to each candidate gene.

3. Seal the plate with plastic film and centrifuge for $1 \mathrm{~min}$ at $300 \times g$ at RT.

4. Place the plate into a qPCR machine and then start the program at $95^{\circ} \mathrm{C}$ for $3 \mathrm{~min}$, followed by $95{ }^{\circ} \mathrm{C}$ for $15 \mathrm{~s}$, and then $55{ }^{\circ} \mathrm{C}$ for $60 \mathrm{~s}$. Repeat the subsequent two steps for 45 cycles.

\section{Representative Results}

The process of EEC and CEC isolation is described in Figure 2. The successful isolation of EECs and CECs was determined by assessing the presence of pan-endothelial cell markers, as well as those distinct to the two subtype populations. As predicted, qPCR revealed that relative to $\beta$-actin, EECs expressed higher levels of endocardial markers Npr3, Hapln1, and Cdh11 compared to CECs (Figure 3A). Likewise, CECs expressed higher levels of coronary markers Fabp4, Mgll, and Cd36 compared to EECs (Figure 3B). Additionally, both EECs and CECs expressed the pan-EC marker gene Cdh5, with slightly higher levels in CEC (Figure 3C). 


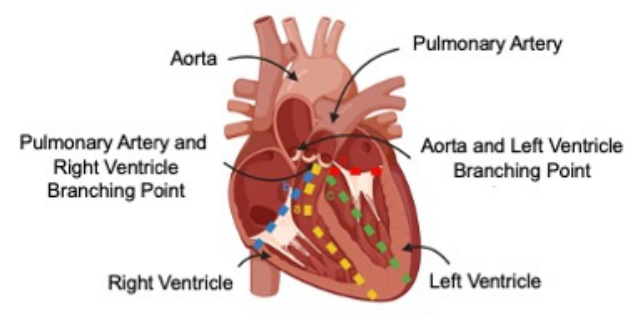

Figure 1: Diagram of heart dissection. (A) First cut made to separate the right ventricle free wall from the septum. (B) Second cut made to liberate the right ventricle completely. (C) First cut made to separate the left ventricle free wall from the septum. (D) Second cut made to liberate the left ventricle completely. Please click here to view a larger version of this figure.

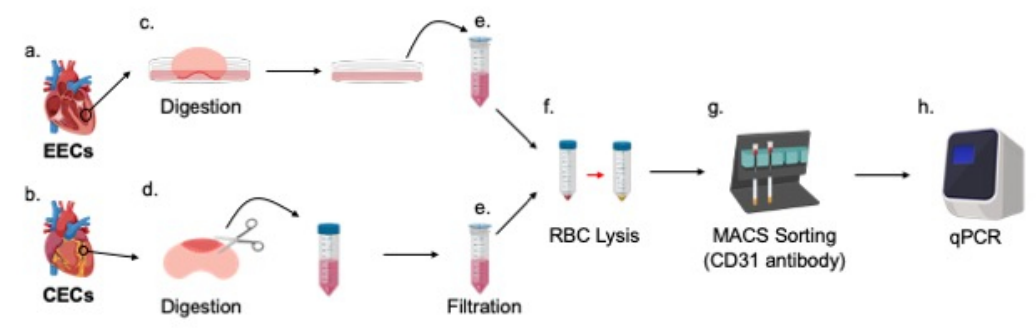

Figure 2: Diagram of digestion set-up of the CECs and EECs and the following arrangement for cell sorting. (A) Innermost free ventricular wall and (B) outermost ventricular free wall were $(\mathbf{C})$ immersed in digestion buffer or (D) digested in digestion buffer respectively. (E) Collection and filtration of cell solutions followed by (F) red blood cell (RBC) lysis and (G) magnetic-activated cell sorting (MACS) using the CD31 antibody. (h) Purified ECs were processed for gene expression verification using qPCR. Please click here to view a larger version of this figure. 
A.

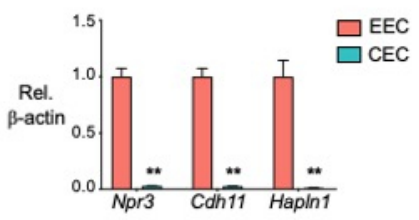

B.

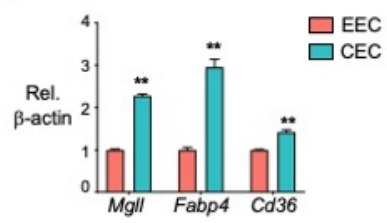

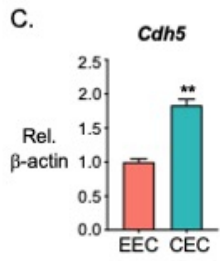

Figure 3: qPCR data verifying isolation of CECs and EECs. (A) Gene expression levels of the EEC markers Npr3, Cdh11, and Hapln1 in EECs and CECs were quantified by real-time PCR. (B) Gene expression levels of the CEC markers Mgll, Fabp4, and Cd36 in EECs and CECs were quantified by real-time PCR. (C) The pan-EC marker Cdh5 was quantified in EECs and CECs by real-time PCR. ( $n=3$ in each group). Bars represent mean \pm SEM. ${ }^{*} p<0.05,{ }^{* *} p<0.01$ vs. EEC, unpaired t-test. Please click here to view a larger version of this figure.

\begin{tabular}{|l|l|l|l|}
\hline Table 1. Digestion Buffer (1.5ml) & Stock Con. & Final Con. & Volume \\
\hline & $5 \mathrm{mg} / \mathrm{ml}$ & $0.5 \mathrm{mg} / \mathrm{ml}$ & $150 \mathrm{ul}$ \\
\hline Liberase TM & $1 \mathrm{mg} / \mathrm{ml}$ & $20 \mathrm{ug} / \mathrm{ml}$ & $30 \mathrm{ul}$ \\
\hline Dnase I & $1 \mathrm{M}$ & $10 \mathrm{mM}$ & $15 \mathrm{ul}$ \\
\hline HEPES & - & - & $1305 \mathrm{ul}$ \\
\hline DMEM & - & & \\
\hline
\end{tabular}

Table 1: Recipe for the digestion buffer.

\begin{tabular}{|l|l|l|}
\hline Table 2. Primer Sequences & Forward & Reverse \\
\hline Gene Name & TCCTTGCAAATCATGTGGCCTA & GGAATCTTCCCGCAGCTCTC \\
\hline Npr3 & GTGAATGGGACTGGGACTGG & GTAATTTCTGGGGCCCTTGC \\
\hline Cdh11 & CCAGCTAAGTGGGACTCGAAG & GGGCCATTTTCAGCTTGGATG \\
\hline Mgll & CCCGGGGCCCAAAGAC & GAAGATGAGGGCCTTGGGTG \\
\hline Fabp4 & AGAAGTGGGAGTTGGCTTCG & ACTCTCTGACCGGATGACGA \\
\hline Cd36 & GCAAAACGACTGCAGGTCAA & CCCGGTCACTTGGTTTCTGA \\
\hline Cdh5 & CCATTGAGACAGACCCCGAC & TGTGGAACGTGTACTGCTGG \\
\hline B-actin & TCTGTGTGGATTGGTGGCTC & CGGACTCATCGTACTCCTGC \\
\hline
\end{tabular}

Table 2: Primer Sequences

\section{Discussion}

CECs and EECs differ in origin, markers, and functions, and thus could play unique roles in development and disease. Existing protocols for endothelial cell isolation are limited to macrovascular tissues, neglecting the collection of EECs, thus restricting the study of CEC- and EECspecific functions. It is essential to isolate and study these two populations independently, as this knowledge would provide a reference for the differentiation of iPSCs into CECs and EECs for future studies and facilitate the examination of these cell populations for potential therapeutic targets for various cardiac diseases. This novel protocol outlines a method for the isolation of EECs from the inner surface and CECs from the outer surface of the ventricular free wall of adult rats.

It is critical to control the timing for each step very precisely in this protocol. Because the number of EECs is very limited in rat heart tissue, we minimized the digestion time of the inner layer of the heart to prevent cellular damage and more importantly, CEC contamination. Immediate addition of the EC medium solution to terminate the enzymatic reaction is also very important for maintaining high cell viability. A red pellet following cell collection suggests the presence of a large number of RBCs. Depending on the amount of RBC contamination, 1-2 mL of RBC lysis buffer can be added to degrade the RBCs. Incubation must be carefully timed to avoid significant damage to the cells, and PBS is promptly 
added to terminate the reaction. Using the current protocol, we were able to isolate $10^{5}$ EECs from six rat hearts. These cells could be seeded onto a cell culture dish for further expansion and characterization.

When preparing the tissue for free wall collection, the heart was washed with HBSS to remove the majority of the remaining RBCs. HBSS is the recommended medium due to its clear appearance, which enables the visualization of blood cells, in contrast to DMED containing phenol red. The composition of the digestion buffer ensures sufficient liberation of endothelial cells, where liberase digestion enzyme strips the tissue of the exposed cells, DNase I eliminates DNA from the dead cells to promote cell detachment that may be inhibited by DNA's adhesive quality, HEPES buffer balances the $\mathrm{pH}$, and DMEM is a modified basal medium with a higher concentration of amino acids and vitamins for better cell maintenance and contains the calcium necessary to activate the liberase.

According to the single cell RNA sequencing (scRNA-seq) obtained from both human ${ }^{15}$ and mouse heart ${ }^{10}$, several markers attributed to specific EC populations were reported. We selected some of the most enriched genes to examine the purity of isolated EECs (i.e., Npr3, Cdh11, and Hapln1) and EECs (i.e., Mgll, Fabp4, and Cd36). Relative to $\beta$-Actin, Npr3, Cdh11, and Hapln1 markers demonstrated increased expression in EECs compared to CECs. Similarly, the expression of Mgll, Fabp4, and Cd36 markers was greater in CECs compared to EECs. The uniquely expressed markers for each sample is in accord with markers characteristic to EECs and CECs respectively, indicating successful isolation.

However, the current protocol cannot rule out cross contamination between the two EC populations during the isolation, even with carefully controlled sequential digestions. Therefore, some cell surface markers can be applied for further purification. For example, NPR3 generally labels the endocardium ${ }^{10}$, whereas APJ can trace a majority of the CECs ${ }^{16}$. Because these two markers are expressed on the cell surface, they could be used for fluorescence activated cell sorting (FACS), and antibodies used to further purify distinct EC populations. In addition, human ${ }^{15}$ and mouse ${ }^{10}$ heart scRNA-seq can confirm enrichment of Npr3 in EECs, and Cd36 can be potentially used for CEC purification.

In conclusion, the presented protocol outlines the independent isolation of EECs and CECs from the rat heart. The comprehensive identification of cellular properties, enabled by cell isolation, can be utilized for significant downstream applications.

\section{Disclosures}

The authors have nothing to disclose.

\section{Acknowledgments}

The authors greatly appreciate Dr. Lingli Wang for her help with the animal protocol, and the Department of Pediatrics at Stanford for infrastructure support. This work was supported by NIH/NHLBI K99 HL135258 (to M.G.).

\section{References}

1. Gladka, M. M. et al. Single-Cell Sequencing of the Healthy and Diseased Heart Reveals Cytoskeleton-Associated Protein 4 as a New Modulator of Fibroblasts Activation. Circulation. 138 (2), 166-180 (2018).

2. Koren, C. W., Sveinbjornsson, B., Smedsrod, B. Isolation and culture of endocardial endothelial cells from Atlantic salmon (Salmo salar) and Atlantic cod (Gadus morhua). Cell and Tissue Research. 290 (1), 89-99 (1997).

3. Tian, X. et al. Subepicardial endothelial cells invade the embryonic ventricle wall to form coronary arteries. Cell Research. 23 (9), 1075-1090 (2013).

4. $\mathrm{Wu}, \mathrm{B}$. et al. Endocardial cells form the coronary arteries by angiogenesis through myocardial-endocardial VEGF signaling. Cell. 151 (5), 1083-1096 (2012).

5. Li, Y., Lui, K. O., Zhou, B. Reassessing endothelial-to-mesenchymal transition in cardiovascular diseases. Nature Reviews in Cardiology. 15 (8), 445-456 (2018).

6. Zhang, H., Lui, K. O., Zhou, B. Endocardial Cell Plasticity in Cardiac Development, Diseases and Regeneration. Circulation Research. 122 (5), 774-789 (2018).

7. Yu, S. Y. et al. Isolation and characterization of human coronary artery-derived endothelial cells in vivo from patients undergoing percutaneous coronary interventions. Journal of Vascular Research. 46 (5), 487-494 (2009).

8. Red-Horse, K., Ueno, H., Weissman, I. L., Krasnow, M. A. Coronary arteries form by developmental reprogramming of venous cells. Nature. 464 (7288), 549-553 (2010).

9. Kamimura, T., Yamagishi, T., Nakajima, Y. Avian coronary endothelium is a mosaic of sinus venosus- and ventricle-derived endothelial cells in a region-specific manner. Development, Growth and Differentiation. 60 (2), 97-111 (2018).

10. Zhang, H. et al. Endocardium Minimally Contributes to Coronary Endothelium in the Embryonic Ventricular Free Walls. Circulation Research. 118 (12), 1880-1893 (2016).

11. Kinlay, S., Libby, P., Ganz, P. Endothelial function and coronary artery disease. Current Opinion in Lipidology. 12 (4), $383-389$ (2001).

12. Jacques, D., Bkaily, G. Endocardial endothelial cell hypertrophy takes place during the development of hereditary cardiomyopathy. Molecular and Cell Biochemistry. 453 (1-2), 157-161 (2019).

13. Grossfeld, P., Nie, S., Lin, L., Wang, L., Anderson, R. H. Hypoplastic Left Heart Syndrome: A New Paradigm for an Old Disease? Journal of Cardiovascular Development and Disease. 6 (1), (2019).

14. Li, Y. et al. Down-regulated RGS5 by genetic variants impairs endothelial cell function and contributes to coronary artery disease. Cardiovascular Research. (2019).

15. Miao, Y. et al. Single-Cell RNA-Seq Reveals Endocardial Defect in Hypoplastic Left Heart Syndrome. bioRxiv. (2019).

16. Chen, H. I. et al. The sinus venosus contributes to coronary vasculature through VEGFC-stimulated angiogenesis. Development. 141 (23), 4500-4512 (2014). 